\title{
Systematic review of spinal and intracranial complications after central neuraxial blocks for perioperative and obstetric anaesthesia and analgesia. Part II: Abscess
}

\author{
Bos E.M.E. ${ }^{1}$, Haumann J. ${ }^{2}$, de Quelerij M. ${ }^{3}$, Kalkman C.J. ${ }^{4}$, Hollmann M.W. ${ }^{1}$, Lirk P.B. ${ }^{5}$
}

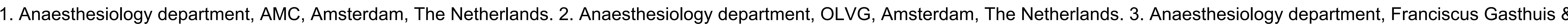
Vlietland, Rotterdam, The Netherlands. 4. Anaesthesiology department, UMC, Utrecht, The Netherlands. 5. Anaesthesiology department, Harvard Medical School, Boston MA, USA

\section{Introduction}

Feared infectious complications of central neuraxial blocks (CNB) are spinal or intracranial abscess. Due to the rarity of these complications, the characteristics of patients, symptoms, patterns of treatment and outcome are not well described.

\section{Methods}

A systematic literature search was performed in three databases (Medline, Embase and the Cochrane Library) for reports of spinal and intracranial abscess associated with CNB. Spinal abscess is defined as epidural, subdural or subarachnoid haematoma in the vertebral canal. Patient characteristics, symptoms, risk factors and neurological outcome were analyzed.

\section{Results}

The search yielded 667 articles, of which 137 articles were reviewed; 253 patients with spinal abscess (SA), 3 patients with intracranial abscess (IA) and 1 patient with both SA and IA were reported. Neurological recovery after treatment was correlated with initial neurological deficit. IA occurred in 3 females; 2 cases after spinal anaesthesia and 1 case after epidural anaesthesia.

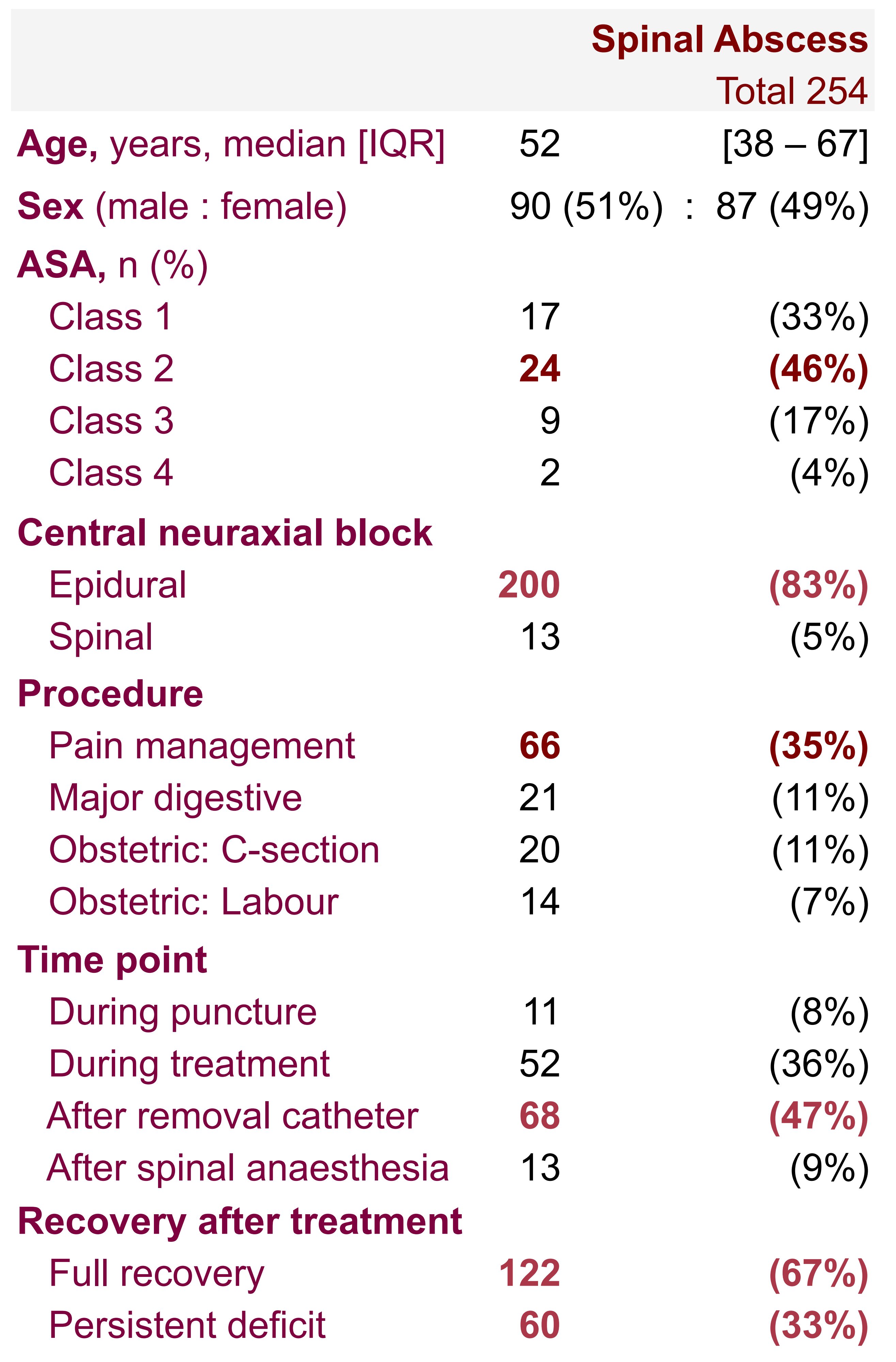

Table 1. Characteristics of patients with spinal abscess.

Sex

\section{Female}

Male

Degree motor deficit**

No / Mild

Severe / Full

Degree not specified

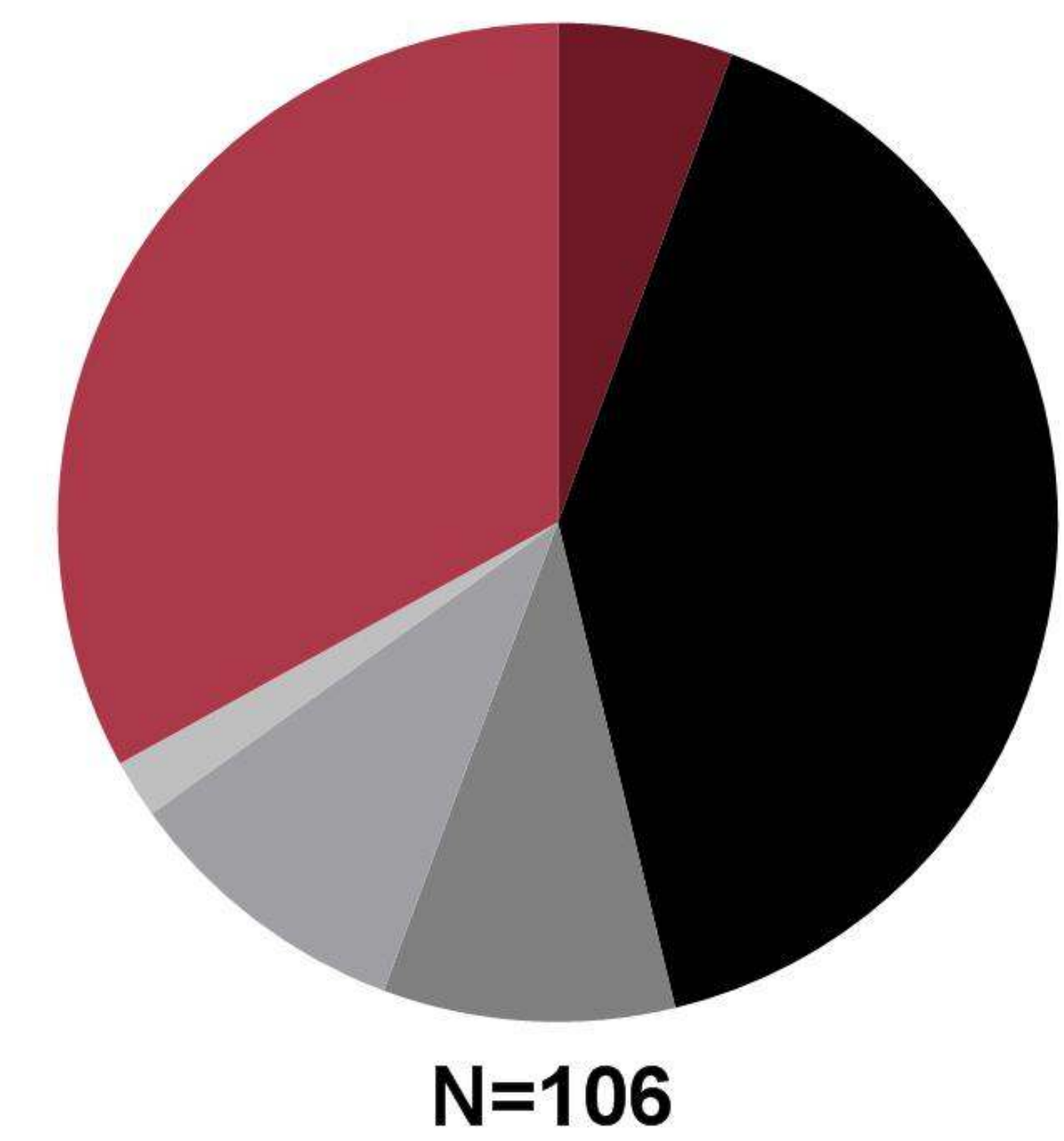

- No Symptoms $5.7 \%$

- Pain: isolated symptom $\mathbf{4 0 . 6 \%}$

Pain and Sensory deficit $9.4 \%$

- Pain and Motor deficit $9.4 \%$

- Sensory and Motor deficit $1.9 \%$

- Pain, Sensory and Motor deficit 33.0\%
$(46 \%)$

\section{Spinal Abscess \\ aOR (95\% C.I.) $\quad P$}

Timing of neurosurgical decompression from symptom (hours). No significant difference were encountered between timing of decompression from symptom onset between patients with full recovery and patients with partial or no recovery $(P: 0.264)$.
Spinal Abscess

Full recovery

Partial / No recovery
.01

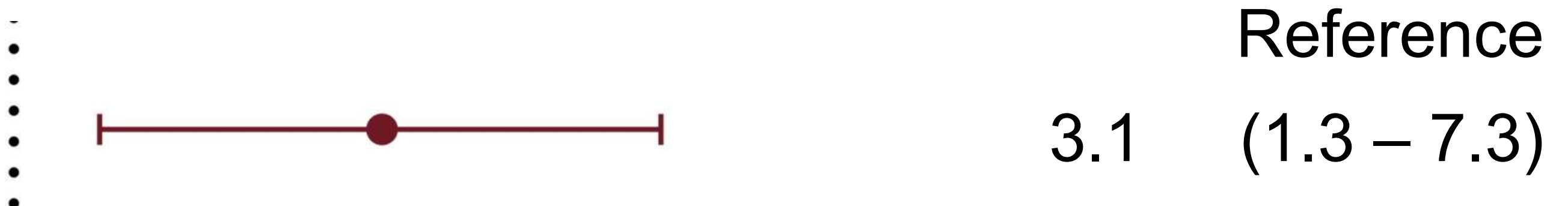

Reference

$3.7 \quad(1.2-11.4)$

$1.0 \quad(0.3-2.8)$
Table 2. Adjusted Odds ratios (aOR) for persistent neurological deficit in patients with neurological deficit prior to treatment, data is presented for multivariate regression analysis of multiple imputed data. $\mathrm{N}=149$ cases. Adjusted for sex and degree of motor deficit.

\section{Discussion \& Conclusion}

Patients can experience spinal or intracranial abscess after CNB. Caution should be taken when interpreting the results of this review, since publication bias, selection bias and underreporting of complications may have influenced the results, however we believe that the information of this review is helpful in understanding rare severe complications of CNB. Based on the results we can conclude that abscesses associated with CNB often occur when patients are out of the scope of the anaesthesiologists work field. In patients with pain as isolated symptom, conservative management may be indicated, provided that continuous patient monitoring is guaranteed. Since diverse details were encountered during analysis, we want to accentuate that uniform registries are needed to identify relevant risk factors and to establish a more accurate estimate of the incidence of serious complications of CNB. 